\title{
A new synthetic approach to 3-methylene-1-cyclohexanols fused to five-, six- and seven-membered carbocycles through intramolecular cyclization of epoxyallylsilanes
}

\author{
Asunción Barbero, Pilar Castreño, Francisco J. Pulido*, Patricia Val, Alfonso González- \\ Ortega, and M. Carmen Sañudo \\ Departamento de Química Orgánica, Facultad de Ciencias, Universidad de Valladolid, \\ Valladolid 47011 Spain \\ E-mail:pulido@qo.uva.es
}

Dedicated to Professor Benito Alcaide on occasion of his $60^{\text {th }}$ birthday

\begin{abstract}
Silylcupration of allene followed by conjugate addition to enones and carbonyl epoxidation provides a simple and high yielding route to epoxyallylsilanes carrying the useful phenyldimethylsilyl group. Lewis acid catalyzed intramolecular cyclization of epoxyallylsilanes is a powerful strategy for carbocyclic annulation of much potential in synthesis. In this article we show A general procedure for the synthesis of 3-methylene-1-cyclohexanols fused to five, six and seven-membered carbocycles by intramolecular cyclization of epoxyallylsilanes, which might be of great interest as an approach to bicyclic systems contained in many naturally occurring products, is described.
\end{abstract}

Keywords: Allylsilanes, allenes, silylcupration, epoxyallylsilanes, intramolecular cyclization, methylenecyclohexanols

\section{Introduction}

Organosilicon compounds and in particular allylsilane chemistry have attracted a great deal of attention due to the increasing number of new methodologies that allow useful synthetic transformations. ${ }^{1}$ Over the last decade allenes have emerged as one of the best sources for the synthesis of allylsilanes. ${ }^{2}$ They are readily attacked by silylcuprates giving rise to a great variety of allylsilanes with different substitution patterns. ${ }^{3}$ The stoichiometry of the silylcuprate (higher or lower order) is responsible for the final regioselectivity of the reaction, leading selectively to allylsilanes when a lower order cyanosilylcuprate $\left(\mathrm{R}_{3} \mathrm{SiCuCNLi}\right)$ is used. ${ }^{4}$ 


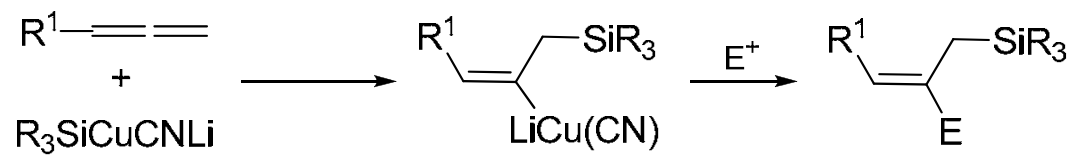

\section{Scheme 1}

$\alpha, \beta$-Unsaturated oxocompounds have been successfully used in this reaction, leading to a wide range of oxo-functionalized allylsilanes, which are valuable intermediates for carbocyclic annulations. ${ }^{4,5}$ In recent reviews, we have shown a general summary of the advances in allylsilane chemistry and their significance as precursors for the synthesis of three to sevenmembered rings. ${ }^{2 b, 6}$

Despite its synthetic potential, the cyclization of epoxyallylsilanes has not been widely explored. Under Lewis acid conditions, nucleophilic substitution usually takes place at the most substituted carbon center of the epoxide ${ }^{7}$ unless the presence of electron-withdrawing groups next to the epoxide destabilizes the developing carbocation. ${ }^{8}$ Contrary to the normal 5-exo ${ }^{9}$ or 6 endo ${ }^{10,11}$ attack, we have recently reported that the acid-catalyzed reaction of epoxyallylsilanes containing the phenyldimethylsilyl ${ }^{12}$ or the tert-butyldiphenylsilyl ${ }^{13,14}$ group follows an unusual rearrangement-cyclization process which leads to methylenecyclohexanols ${ }^{12}$ or to allyl- and vinylsilane-containing cyclohexanols ${ }^{13}$ respectively (Scheme 2 ).
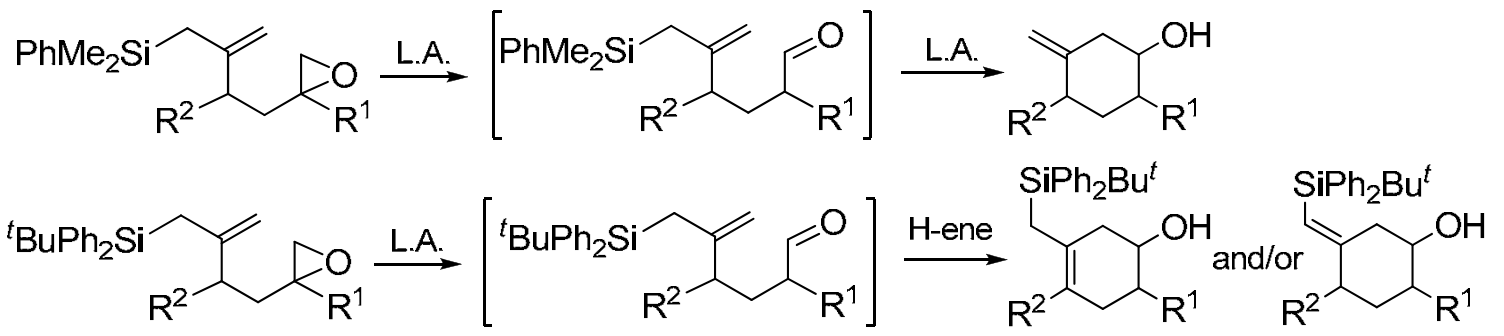

\section{Scheme 2}

\section{Results and Discussion}

Epoxyallylsilanes have been previously prepared by Wittig reaction of an aldehyde with $\mathrm{Ph}_{3} \mathrm{P}=\mathrm{CHCH}_{2} \mathrm{SiMe}_{3}$ followed by epoxidation ${ }^{15}$ and by cross metathesis of alkenyl epoxides and allylsilanes. ${ }^{16}$ We have recently reported ${ }^{12}$ a new route for the synthesis of epoxyallylsilanes by silylcupration of allene, followed by capture of the intermediate cuprate with enones and finally sulfur ylide-mediated epoxidation.

In the past years, we have been very actively involved in the study of the silyl- and stannylcupration of allenes and acetylenes and their synthetic applications. ${ }^{2 a, 17}$ These reactions take place by syn-addition of copper to one end of the multiple bond and Si or Sn to the other, 
affording cuprates with structures comparable to $\mathbf{1}$. The intermediate allylsilane-vinylcuprate $\mathbf{1}$ can be captured by a great variety of electrophiles giving functionalized allylsilanes. ${ }^{2 a}$ For example, the use of $\alpha, \beta$-unsaturated oxocompounds leads to oxoallylsilanes of type $\mathbf{2}$ which have been used as powerful building blocks for cyclopentane annelations. ${ }^{2 a, 6 a, 18}$ Epoxidation of $\mathbf{2}$ via dimethylsulfonium-ylides gives epoxyallylsilanes $\mathbf{3}$ in high yield (Scheme 3). ${ }^{12,13}$

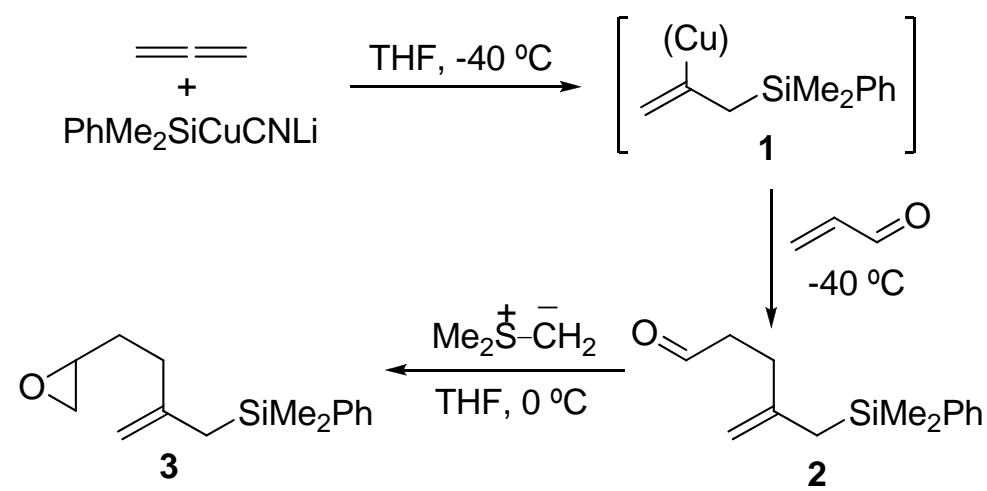

\section{Scheme 3}

In this paper we describe the synthesis and cyclization of epoxyallylsilanes containing five-, six- and seven-membered carbocycles as an efficient route for the construction of bicyclic 3methylene-1-cyclohexanols systems which are present in the skeleton of many terpenoid products. As it is shown, Lewis acid-catalyzed intramolecular cyclization seems to occur with concomitant rearrangement of the epoxy group and trapping of the intermediate aldehyde (Table 2). ${ }^{19}$

Phenyldimethylsilylcyanocuprate ${ }^{20}$ reacts with allene at $-40^{\circ} \mathrm{C}$ giving an allylsilanevinylcopper intermediate $\mathbf{1}$, which was treated with cyclic $\alpha, \beta$-unsaturated ketones 4-8 to afford the products of conjugate addition 9-14 in good yield. All the reactions were carried out in the presence of $\mathrm{BF}_{3} \cdot \mathrm{OEt}_{2}$ or $\mathrm{TMSCl}$, which considerably increased the yield (Table 1). The cyclic oxoallylsilanes thus obtained were treated with dimethylsulfonium methylide to give in good yield the corresponding epoxides 15-20 (Table 1) apparently as a single diastereomer (single sharp signals in ${ }^{13} \mathrm{C}$ NMR spectra). ${ }^{21}$ No attempt was made to assign configurations to the epoxides. 
Table 1. Synthesis of oxo- and epoxyallylsilanes.

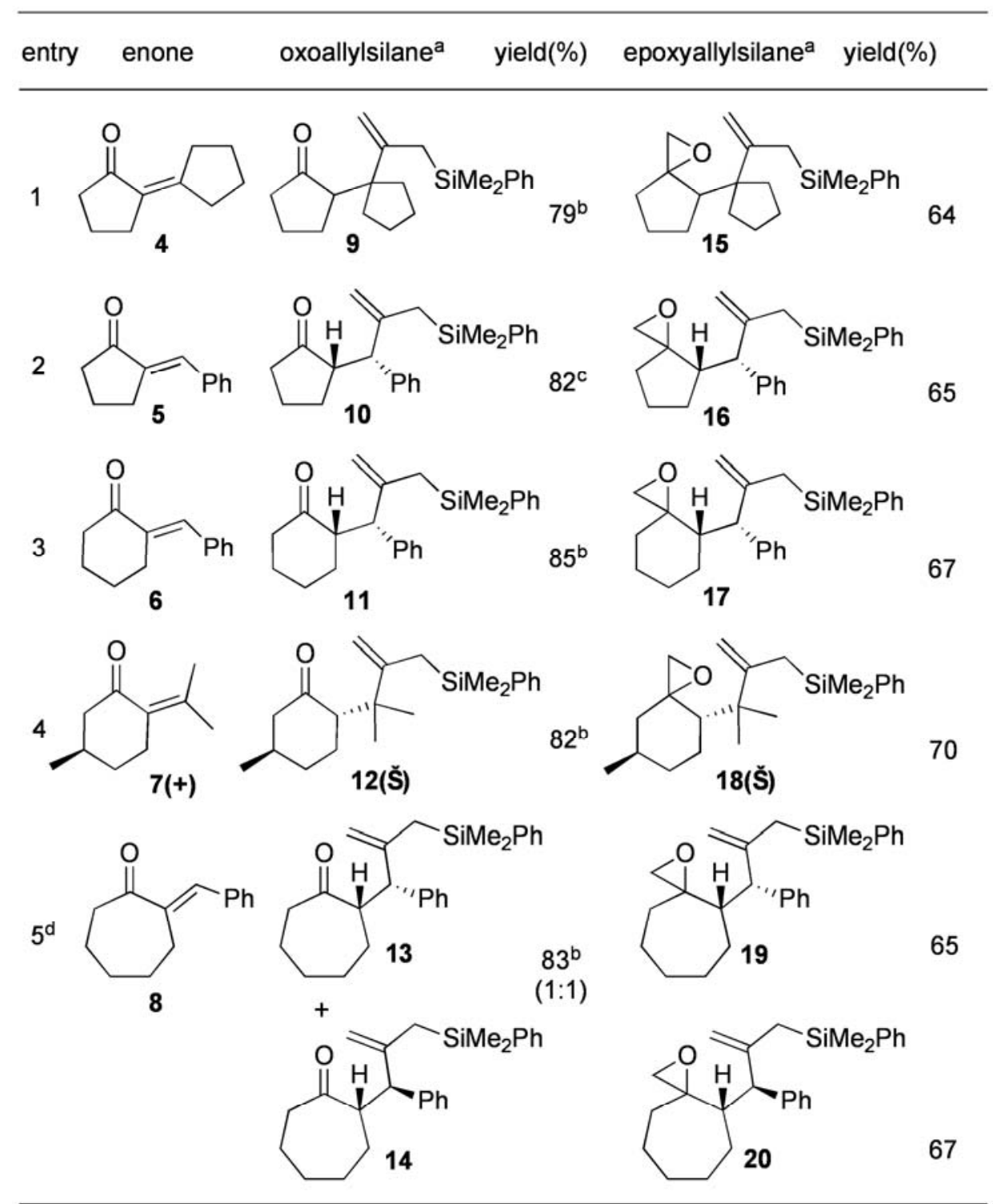

a THF was used as solvent. ${ }^{\mathrm{b}} \mathrm{BF}_{3}$. $\mathrm{Et}_{2} \mathrm{O}$ was use as activant. ${ }^{\mathrm{c}} \mathrm{TMSCl}$ was use as activant. ${ }^{\mathrm{d} C o m p o u n d s} \mathbf{1 3}$ and $\mathbf{1 4}$ separated by LC (ratio $1: 1$ ).

The stereochemistry of the isolated compound was established by COSY and NOESY spectra, and by examining their ${ }^{1} \mathrm{H}$ coupling constants. For the entry 5, both diastereomers 13 and 14 could be well separated by LC, and their relative stereochemistry assigned. Both were efficiently epoxidated with retention of their initial configuration. 
The most remarkable feature observed in the epoxyallylsilane cyclization is the absence of products resulting from 5-exo or 6-endo cyclization (the normal epoxide cleavage). Instead, we selectively obtained bicyclic 3-methylene-1-cyclohexanols 21-29 derived from an unusual rearrangement-cyclization process (Table 2). The rearrangement of a carbonyl group into an epoxide is known process that is enhanced by the presence of acids. ${ }^{22}$ However, this is the first time that this sequential tandem reaction is observed in epoxyallylsilanes. The substituents on the silyl group should play an important role because epoxyallylsilanes carrying TMS groups give normal products of 5-exo or 6-endo attack. ${ }^{11}$

The results collected in Table 2 seems to indicate that in the case of epoxyallylsilanes bearing $\mathrm{PhMe}_{2} \mathrm{Si}$ groups, rearrangement occurs faster than the nucleophilic substitution on the epoxy group. A two-step mechanism involving rearrangement to intermediate A followed by intramolecular Sakurai-Hosomi cyclization has been proposed (Table 2). The second step (cyclization) must be a fast reaction since aldehyde intermediates of type A never were isolated. To optimize the procedure, several combinations of solvent, temperature, time and Lewis acid were carefully tested. Boron trifluoride etherate in dichloromethane was proven to be the best choice for good yield (table 2). From a synthetic point of view, the reported methodology is highly attractive because it provides an efficient strategy for the construction of bicyclic 3methylene-1-cyclohexanols fused to five-, six- and seven-membered ring systems contained in many natural products. 
Table 2.

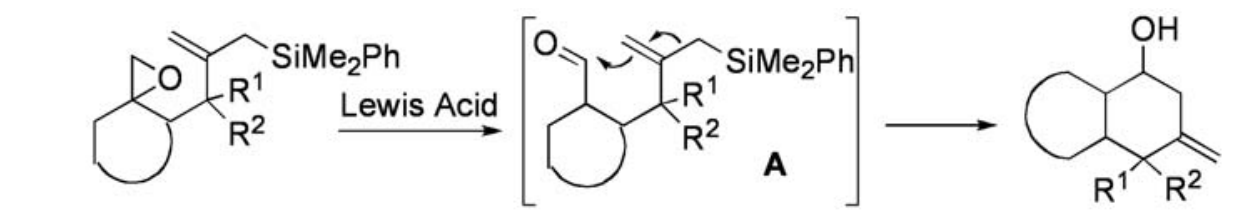

\begin{tabular}{|c|c|c|c|c|c|}
\hline Entry & Epoxiallylsilane & $\begin{array}{l}\text { Lewis Acid }^{\mathrm{a}} \\
\text { solvent }\end{array}$ & $\begin{array}{c}\mathrm{T}(\tilde{\mathbb{C}}) \\
\text { react. time (min) }\end{array}$ & $\begin{array}{c}\text { Methylenecyclohexanols } \\
{\text { (ratio })^{\mathrm{b}}}\end{array}$ & $\begin{array}{l}\text { Yield } \\
(\%)^{c}\end{array}$ \\
\hline
\end{tabular}

1<smiles>C=C(CCc1ccccc1)C1(C2CCCC2)CCC23CCCC2C31CCC</smiles>

2<smiles>C=C(CSc1ccccc1)C(c1ccccc1)C1CCCC12CO2</smiles>

3<smiles>C=C(CSc1ccccc1)[C@H](c1ccccc1)C1CCCCC12CO2</smiles>
17<smiles>C=C(CCc1ccccc1)C(C)(C)[C@H]1CCC(C)CC12CC2(C)[Mg]</smiles>

18(S)

5<smiles></smiles>

19<smiles>C=C(CSc1ccccc1)C(C(c1ccccc1)c1ccccc1)C1(O)CCCCCC12CO2</smiles>

$\begin{array}{lc}\mathrm{BF}_{3} \cdot \mathrm{OEt}_{2} & 0 \\ \mathrm{DCM} & 40\end{array}$

$\begin{array}{lc}\mathrm{BF}_{3} \cdot \mathrm{OEt}_{2} & 0 \\ \mathrm{DCM} & 40\end{array}$<smiles>C=C1C[C@H](O)[C@@H]2CCC[C@H]2C12CCCC2</smiles>

89 21

85<smiles>C=C1C[C@H](O)[C@@H]2CCC[C@@H]2[C@H]1c1ccccc1</smiles><smiles>C=C1C[C@@H](c2ccccc2)C(=C)C[C@H](O)C2CCCC[C@H]2C1c1ccccc1</smiles>

88

88

$\begin{array}{lc}\mathrm{BF}_{3} \cdot \mathrm{OEt}_{2} & 0 \\ \mathrm{DCM} & 40\end{array}$<smiles>C=C1C[C@@H](O)C2C[C@@H](C)CCC2C(C)(C)C1(C)C</smiles>

25(+) $\begin{array}{lc}\mathrm{BF}_{3} \cdot \mathrm{OEt}_{2} & 0 \\ \mathrm{DCM} & 40\end{array}$<smiles>C=C(C)C1CC(O)C2CCCCCC2C1c1ccccc1</smiles>

80

75

$\begin{array}{lc}\mathrm{BF}_{3} \cdot \mathrm{OEt}_{2} & 0 \\ \mathrm{DCM} & 40\end{array}$<smiles>C=C1C[C@H](O)C2CCCCCC23CCCCCC3[C@H]1c1ccccc1</smiles>

1:1

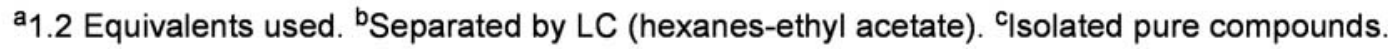

High levels of stereoselectivity were found in the formation and cyclization of epoxyallylsilanes. Except for compound 20, all epoxyallylsilane cyclizations afford selectively cis-fused bicyclic systems. Moreover, formation of 26 and 27 seems to point out that, in some cases, the loss of proton competes favorably with the leaving of silyl group. 
The cis stereochemistry of fused rings and the configurations of the phenyl and hydroxyl bearing carbons were assigned by examining their COSY and NOESY spectra. For example, in compound 23 observation of coupling patterns for $\mathrm{H}(1) \delta=4.1 \mathrm{ppm}$ and $\mathrm{H}(4) \delta=3.53$ ppm clearly indicates that they are axial $(\mathrm{OH} / \mathrm{Ph}$, trans di- equatorial). The former protons also have clear COSY correlations with $\mathrm{H}(8 \mathrm{a}) \delta=1.86 \mathrm{ppm}$ and $\mathrm{H}(4 \mathrm{a}) \delta=1.95 \mathrm{ppm}$ respectively. Examination of the NOE correlations between $\mathrm{H}(4 \mathrm{a})$ and $\mathrm{H}(8 \mathrm{a})$ shows strong cross-peaks at the coordinates, which support cis-fused stereochemistry. $\mathrm{H}(4 \mathrm{a})$ also has an intense NOE correlation with $\mathrm{H}(4)$ which suggest that both protons are in a syn relationship $(\mathrm{Ph} / \mathrm{H}(4 \mathrm{a})$ : anti). Finally, no cross-peaks were found for the correlation $\mathrm{H}(1)-\mathrm{H}(8 \mathrm{a})$, however syn-protons $\mathrm{H}(1)$ and $\mathrm{H}_{\mathrm{eq}}(2) \delta=2.23 \mathrm{ppm}$ show NOE correlation, thus confirming the anti relationship of $\mathrm{H}(1)$ and $\mathrm{H}(8 \mathrm{a})$.

As it is shown in the example of Scheme 4, addition of cuprate $\mathbf{1}$ to the enone $\mathbf{6}$ is followed by stereoselective protonation from the less hindered side of the enolate to give selectively oxoallylsilane 11 (8:1, Table 1). The epoxide 17 undergoes rearrangement, in the presence of boron trifluoride etherate, probably through a cationic intermediary (Scheme 4) to give an intermediate aldehyde A (not isolated). Subsequent cyclization to bicyclic methylenecyclohexanol 23 takes place via a low-energy transition state B (Scheme 4) where the bulky groups attain an equatorial conformation for minimal steric repulsions. The cis-fused ring pattern obtained in most cases might indicate that rearrangement give just the intermediate aldehyde $\mathbf{A}$ of cis stereochemistry, however, it is also feasible that the two possible $\alpha$-epimers of the aldehyde A equilibrate, with the one which can cyclize to give cis-fused products reacting faster and therefore giving rise to the final products.
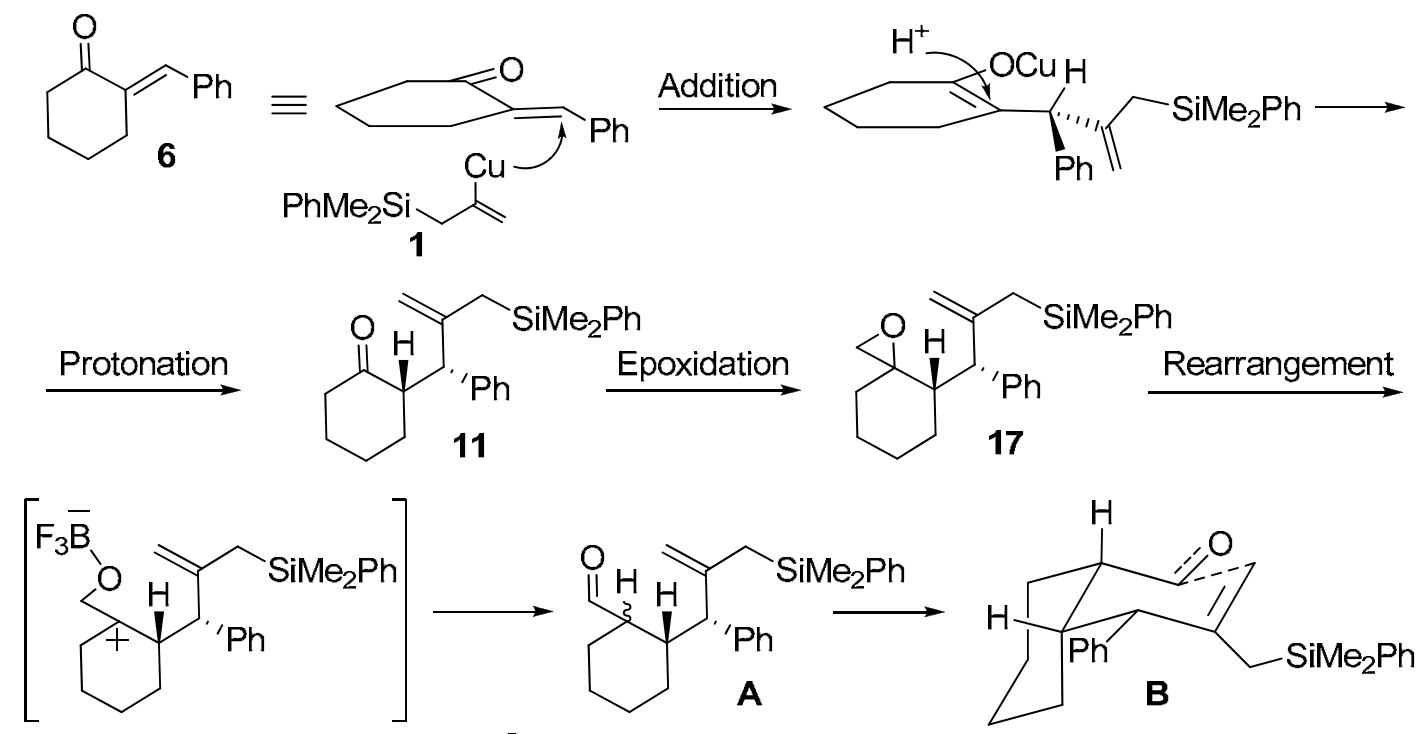<smiles>C=C1C[C@H](O)[C@H](c2ccccc2)[C@H]2CCCC[C@H]12</smiles>

\section{Scheme 4}


In summary, a new route to methylenecyclohexanols fused to five, six and seven-membered carbocyclic rings via epoxyallylsilane cyclization is described. The stereoselectivity of the reaction allows the efficient preparation of 5/6,6/6 and 7/6 cis-fused bicyclic systems contained in many natural products. An unusual rearrangement-cyclization tandem process has been proposed as a feasible mechanism for the general reaction reported.

\section{Experimental Section}

General Procedures. Organometallic reagents were purchased from Aldrich and $\mathrm{CuCN}$ from Fluka. The latter was briefly dried in vacuo prior to use. 1,2-Propadiene (Allene) was supplied by Air Liquide S. A. in lecture bottles. All reactions were carried out under dry nitrogen. Elemental analyses were performed with a LECO CHNS-932 analyzer sited in the "Parque Cientifico Tecnológico de la UBU, Pza. Misael Bañuelos s/n, 09001 Burgos Spain”. The stereochemistry of compounds has been assigned on the basis of COSY and NOESY experiments.

\section{Silylcupration of allene. General procedure ${ }^{4}$}

A solution of phenyldimethylsilyl-lithium ${ }^{23}(3 \mathrm{mmol})$, prepared in THF $(3 \mathrm{ml})$ from phenyldimethylchlorosilane and lithium shots $\left(0{ }^{\circ} \mathrm{C}\right.$ to r.t. overnight $)$, was added by syringe to a stirred suspension of copper(I) cyanide $(269 \mathrm{mg}, 3 \mathrm{mmol})$ in THF $(5 \mathrm{ml})$ at $0{ }^{\circ} \mathrm{C}$, under argon atmosphere. The resulting black mixture was stirred at this temperature for $30 \mathrm{~min}$, and then used immediately. The solution of the phenyldimethylsilylcyanocuprate ${ }^{20}(3 \mathrm{mmol})$ in THF $(8 \mathrm{ml})$ was cooled at $-40{ }^{\circ} \mathrm{C}$ and a slight excess of allene was added from a balloon. The mixture was stirred for $1 \mathrm{~h}$ at this temperature and the reagent $\mathbf{1}$ is ready to use. The intermediate cuprates resulting from addition to allene are stable enough in solution to be used without special precautions other than dry atmosphere, however long periods of times before using the reagent are not recommended.

Reaction of the intermediate cuprate 1 with enones 4-8. Typical procedure ${ }^{4} \mathrm{BF}_{3} \cdot \mathrm{Et}_{2} \mathrm{O}$ $(0.38 \mathrm{ml}, 3 \mathrm{mmol})$ was added at $-78{ }^{\circ} \mathrm{C}$ to a stirred solution of the intermediate cuprate $\mathbf{1}$ ( $3 \mathrm{mmol}$ ) and the mixture stirred for $10 \mathrm{~min}$ at this temperature, then $3.5 \mathrm{mmol}$ of the $\alpha, \beta$ unsaturated oxocompound 4-8 in THF $(5 \mathrm{ml})$ were added dropwise at $-40{ }^{\circ} \mathrm{C}$ and the resulting mixture was kept at this temperature for $1 \mathrm{~h}$. After gentle warming to $0{ }^{\circ} \mathrm{C}$ (over $0.5 \mathrm{~h}$ ) the mixture was quenched with aq sat. $\mathrm{NH}_{4} \mathrm{Cl}$ solution and extracted with $\mathrm{Et}_{2} \mathrm{O}$. The organic phase was dried, evaporated and chromatographed (EtOAc:hexanes) to give the corresponding oxoallylsilanes 9-14 (Table 1).

1'-(3-Phenyldimethylsilylprop-1-en-2-yl)bi(cyclopentan)-2-one (9). Viscous colorless oil, (79\%); IR (neat): $1741 \mathrm{~cm}^{-1} ;{ }^{1} \mathrm{H} \mathrm{NMR}\left(\mathrm{CDCl}_{3}, 300 \mathrm{MHz}, \delta\right): 7.58-7.35(\mathrm{~m}, 5 \mathrm{H}), 4.81(\mathrm{~s}, 1 \mathrm{H})$, $4.78(\mathrm{~s}, 1 \mathrm{H}), 2.30-1.48(\mathrm{~m}, 15 \mathrm{H}), 1.75(\mathrm{~d}, J=13.5 \mathrm{~Hz}, 1 \mathrm{H}), 1.68(\mathrm{~d}, J=13.5 \mathrm{~Hz}, 1 \mathrm{H}), 0.38(\mathrm{~s}$, $\left.3 \mathrm{H}, \mathrm{CH}_{3} \mathrm{Si}\right), 0.37$ (s, 3H, $\left.\mathrm{CH}_{3} \mathrm{Si}\right) ;{ }^{13} \mathrm{C} \mathrm{NMR}\left(\mathrm{CDCl}_{3}, 75 \mathrm{MHz}, \delta\right): 221.2,148.9,139.5,133.6$, 
$128.9,127.7,110.5,55.5,52.4,40.9,34.1,32.2,27.5,23.4,23.2,21.0,20.2$, -2.1; Anal. Calcd. for $\mathrm{C}_{21} \mathrm{H}_{30} \mathrm{OSi}$ : C, 77.24; H, 9.26. Found: C, 77.55; H, 9.54 .

[2R*,1'R*]-2-(1'-Phenyl-2'-phenyldimethylsilylmethyl-2'-propen-1'-yl)cyclo- pentan-1-one (10). Colorless oil, (82\%); IR (neat): 1737, $1629 \mathrm{~cm}^{-1} ;{ }^{1} \mathrm{H} \mathrm{NMR}\left(\mathrm{CDCl}_{3}, 300 \mathrm{MHz}, \delta\right)$ : 7.70-7.15 $(\mathrm{m}, 10 \mathrm{H}), 4.89(\mathrm{~s}, 1 \mathrm{H}), 4.83(\mathrm{~s}, 1 \mathrm{H}), 3.51(\mathrm{~d}, J=6.7 \mathrm{~Hz}, 1 \mathrm{H}), 2.66(\mathrm{q}, J=8.2 \mathrm{~Hz}, 1 \mathrm{H}), 2.35-2.25$ (m, 1H), 2.19-2.09 (m, 2H), 1.98-1.80 (m, 3H), 1.74 (d, J = 14.3 Hz, 1H), 1.67 (d, J=14.3 Hz, $1 \mathrm{H}), 0.44\left(\mathrm{~s}, 3 \mathrm{H}, \mathrm{CH}_{3} \mathrm{Si}\right), 0.41\left(\mathrm{~s}, 3 \mathrm{H}, \mathrm{CH}_{3} \mathrm{Si}\right) ;{ }^{13} \mathrm{C} \mathrm{NMR}\left(\mathrm{CDCl}_{3}, 75 \mathrm{MHz}, \delta\right): 218.8,146.8$, $141.9,138.9,133.9,129.1,128.9,128.2,127.9,126.5,110.5,52.6,52.2,38.5,28.3,25.5,20.3$, 2.5, -3.1; Anal. Calcd. for $\mathrm{C}_{23} \mathrm{H}_{28} \mathrm{OSi}$ : C, 79.26; H, 8.10. Found: C, 79.60; H, 8.38.

[2R*,1' $\left.R^{*}\right]$-2-(1'-Phenyl-2'-phenyldimethylsilylmethyl-2'-propen-1'-yl)cyclohexan -1-one (11). Colorless oil, (85\%); IR (neat): $1725 \mathrm{~cm}^{-1} ;{ }^{1} \mathrm{H} \mathrm{NMR}\left(\mathrm{CDCl}_{3}, 300 \mathrm{MHz}, \delta\right)$ : $7.51-7.14$ (m, $10 \mathrm{H}), 4.87(\mathrm{~s}, 1 \mathrm{H}), 4.71(\mathrm{~s}, 1 \mathrm{H}), 3.53(\mathrm{~d}, J=10.8 \mathrm{~Hz}, 1 \mathrm{H}), 3.09-3.01(\mathrm{~m}, 1 \mathrm{H}), 2.26-2.21(\mathrm{~m}, 2 \mathrm{H})$, 2.12-2.04 (m, 1H), 1.94-1.89 (m, 1H), 1.81-1.70 (m, 2H), $1.66(\mathrm{~d}, J=14.5 \mathrm{~Hz}, 1 \mathrm{H}), 1.65-1.57$ $(\mathrm{m}, 2 \mathrm{H}), 1.52(\mathrm{~d}, J=14.5 \mathrm{~Hz}, 1 \mathrm{H}), 0.21\left(\mathrm{~s}, 3 \mathrm{H}, \mathrm{CH}_{3} \mathrm{Si}\right), 0.16\left(\mathrm{~s}, 3 \mathrm{H}, \mathrm{CH}_{3} \mathrm{Si}\right) ;{ }^{13} \mathrm{C} \mathrm{NMR}\left(\mathrm{CDCl}_{3}\right.$, $75 \mathrm{MHz}, \delta): 211.8,146.6,142.0,139.1,133.7,129.1,128.5,128.2,127.9,126.4,110.2,53.1$, 52.9, 42.4, 32.8, 29.0, 24.2, 23.1, -2.4, -2.8; Anal. Calcd. for $\mathrm{C}_{24} \mathrm{H}_{30} \mathrm{OSi}$ : C, 79.50; H, 8.34. Found: C, 79.87; H, 8.65.

[2S*,5R*]-2-(3'-Phenyldimethylsilylmethyl-2'-methylbut-3'-en-2-yl)-5-

methylcyclohexanone (12). Colorless oil, $(82 \%)$. $[\alpha]^{20} \mathrm{D}-7.1$ (c $0.88, \mathrm{HCCl}_{3}$ ); IR (neat): 1712, $1630 \mathrm{~cm}^{-1} ;{ }^{1} \mathrm{H} \mathrm{NMR}\left(\mathrm{CDCl}_{3}, 300 \mathrm{MHz}, \delta\right): 7.58-7.35$ (m, 5H), 4.78 (s, 1H), 4.69 (s, 1H), 2.46 $(\mathrm{dd}, J=12.3$ and $4.4 \mathrm{~Hz}, 1 \mathrm{H}), 2.27(\mathrm{dt}, J=11.6$ and $2.7 \mathrm{~Hz}, 1 \mathrm{H}), 2.0(\mathrm{t}, J=12.3 \mathrm{~Hz}, 1 \mathrm{H}), 1.95-$ $1.77(\mathrm{~m}, 3 \mathrm{H}), 1.70(\mathrm{~d}, J=16.8 \mathrm{~Hz}, 1 \mathrm{H}), 1.68(\mathrm{~d}, J=16.8 \mathrm{~Hz}, 1 \mathrm{H}), 1.38(\mathrm{qd}, J=13.0$ and $2.7 \mathrm{~Hz}$, $1 \mathrm{H}), 1.25$ (qd, $J=13.0$ and $2.5 \mathrm{~Hz}, 1 \mathrm{H}), 1.15(\mathrm{~s}, 3 \mathrm{H}), 1.13(\mathrm{~s}, 3 \mathrm{H}), 0.99$ (d, $J=6.1 \mathrm{~Hz}, 3 \mathrm{H}), 0.39$ $\left(\mathrm{s}, 3 \mathrm{H}, \mathrm{CH}_{3} \mathrm{Si}\right), 0.35\left(\mathrm{~s}, 3 \mathrm{H}, \mathrm{CH}_{3} \mathrm{Si}\right) ;{ }^{13} \mathrm{C} \mathrm{NMR}\left(\mathrm{CDCl}_{3}, 75 \mathrm{MHz}, \delta\right): 211.6,152.7,139.7,133.6$, 128.9, 127.6, 109.1, 55.0, 52.4, 41.0, 36.1, 34.6, 28.9, 24.4, 22.3, 21.9, 19.5, -2.0, -2.2; Anal. Calcd. for $\mathrm{C}_{21} \mathrm{H}_{32} \mathrm{OSi}$ : C, 76.77; H, 9.82. Found: C, 77.01; H, 9.99.

[2R*,1'R*]-2-(1'-Phenyl-2'-phenyldimethylsilylmethyl-2'-propen-1'-yl)cycloheptan-1-one (13). Colorless oil, (41.5\%); IR (neat): $1720 \mathrm{~cm}^{-1} ;{ }^{1} \mathrm{H} \mathrm{NMR}\left(\mathrm{CDCl}_{3}, 300 \mathrm{MHz}, \delta\right): 7.62-7.19$ (m, $10 \mathrm{H}), 5.02(\mathrm{~s}, 1 \mathrm{H}), 4.86(\mathrm{~s}, 1 \mathrm{H}), 3.46(\mathrm{~d}, J=10.8 \mathrm{~Hz}, 1 \mathrm{H}), 3.30(\mathrm{td}, J=10.8$ and $3.1 \mathrm{~Hz}, 1 \mathrm{H})$, 2.22-2.05 (m, 3H), 1.95-1.75 (m, 4H), 1.76(d, $J=14.3 \mathrm{~Hz}, 1 \mathrm{H}), 1.65(\mathrm{~d}, J=14.3 \mathrm{~Hz}, 1 \mathrm{H}), 1.40-$ $1.10(\mathrm{~m}, 3 \mathrm{H}), 0.36$ (s, 3H, CH $\left.\mathrm{CH}_{3} \mathrm{Si}\right), 0.34$ (s, 3H, $\left.\mathrm{CH}_{3} \mathrm{Si}\right) ;{ }^{13} \mathrm{C} \mathrm{NMR}\left(\mathrm{CDCl}_{3}, 75 \mathrm{MHz}, \delta\right): 213.7$, $146.4,141.4,138.6,133.4,128.8,128.1,127.8,127.5,126.1,108.7,54.2,53.9,42.9,30.2,28.3$, 28.1, 24.2, 23.7, -2.7, -3.3; Anal. Calcd. for $\mathrm{C}_{25} \mathrm{H}_{32} \mathrm{OSi}$ : C, 79.73; H, 8.56. Found: C, 80.09; H, 8.86 .

[2R*,1'S*]-2-(1'-Phenyl-2'-phenyldimethylsilylmethyl-2'-propen-1'-yl)cyclo- heptan-1-one (14). Colorless oil, (41.5\%); IR (neat): $1716 \mathrm{~cm}^{-1} ;{ }^{1} \mathrm{H} \mathrm{NMR}\left(\mathrm{CDCl}_{3}, 300 \mathrm{MHz}, \delta\right)$ : 7.55-7.05 (m, $10 \mathrm{H}), 4.72(\mathrm{~s}, 1 \mathrm{H}), 4.61(\mathrm{~s}, 1 \mathrm{H}), 3.49(\mathrm{~d}, J=11.0 \mathrm{~Hz}, 1 \mathrm{H}), 3.20(\mathrm{td}, J=11.0$ and $3.1 \mathrm{~Hz}, 1 \mathrm{H})$, $2.62(\mathrm{dt}, J=14.7$ and $3.0 \mathrm{~Hz}, 1 \mathrm{H}), 2.47(\mathrm{ddd}, J=14.7,10.5$ and $5.4 \mathrm{~Hz}, 1 \mathrm{H}), 1.92-1.70(\mathrm{~m}, 2 \mathrm{H})$, $1.66(\mathrm{~d}, J=14.0 \mathrm{~Hz}, 1 \mathrm{H}), 1.52$ (d, $J=14.0 \mathrm{~Hz}, 1 \mathrm{H}), 1.40-1.11(\mathrm{~m}, 4 \mathrm{H}), 1.07-0.85$ (m, $2 \mathrm{H}), 0.41$ $\left(\mathrm{s}, 3 \mathrm{H}, \mathrm{CH}_{3} \mathrm{Si}\right), 0.25$ (s, 3H, $\left.\mathrm{CH}_{3} \mathrm{Si}\right) ;{ }^{13} \mathrm{C} \mathrm{NMR}\left(\mathrm{CDCl}_{3}, 75 \mathrm{MHz}, \delta\right): 214.0,149.2,141.5,139.5$, 
$133.7,129.0,128.7,128.2,127.5,126.5,106.7,54.1,53.7,43.9,30.3,28.9,27.6,26.4,23.4$, 2.6, -3.1 .

\section{Synthesis of epoxyallylsilanes 15-20. Typical procedure ${ }^{12}$}

To a solution of trimethylsulphonium iodide $(1 \mathrm{mmol})$ in dry THF $(5 \mathrm{ml})$ was added dropwise $\mathrm{n}$ BuLi (1 mmol, 1.6 M BuLi in hexanes) and the mixture stirred for $5 \mathrm{~min}$ at $0^{\circ} \mathrm{C}$. Then a solution of the oxoallylsilane 9-14 $(0.8 \mathrm{mmol})$ in THF $(1 \mathrm{ml})$ is added. After stirring for an additional $30 \mathrm{~min}$ at $0^{\circ} \mathrm{C}$ and $1 \mathrm{~h}$ at r.t. brine $(10 \mathrm{ml})$ is added and the mixture extracted with ether, dried and evaporated to dryness. The residue was purified by chromatography to give the corresponding epoxyallylsilanes 15-20 (Table 1).

[2-(1-(1-Oxaspiro[2.4]heptan-4-yl)cyclopentyl)allyl]dimethylphenylsilane (15). Colorless oil, (64\%); IR (neat): $1632 \mathrm{~cm}^{-1} ;{ }^{1} \mathrm{H} \mathrm{NMR}\left(300 \mathrm{MHz}, \mathrm{CDCl}_{3}, \delta\right): 7.60-7.35$ (m, 5H), 4.87 (s, 1H), $4.76(\mathrm{~s}, 1 \mathrm{H}), 2.68(\mathrm{~d}, J=5.6 \mathrm{~Hz}, 1 \mathrm{H}), 2.64(\mathrm{~d}, J=5.6 \mathrm{~Hz}, 1 \mathrm{H}), 2.44(\mathrm{dd}, J=10.0$ and $7.7 \mathrm{~Hz}$, 1H), 1.89-1.40 (m, 16H), $0.38\left(\mathrm{~s}, 6 \mathrm{H},\left(\mathrm{CH}_{3}\right)_{2} \mathrm{Si}\right) ;{ }^{13} \mathrm{C} \mathrm{NMR}\left(\mathrm{CDCl}_{3}, 75 \mathrm{MHz}, \delta\right): 149.3,139.8$, 133.6, 128.8, 127.7, 110.0, 65.6, 55.0, 54.1, 42.7, 35.6, 32.9, 31.4, 30.2, 24.0, 23.5, 22.1, 20.3, 2.0; Anal. Calcd. for $\mathrm{C}_{22} \mathrm{H}_{32} \mathrm{OSi}$ : C, 77.59; H, 9.47. Found: C, 77.90; H, 9.75.

[4R*,1'R*]-4-(1'-Phenyl-2'-phenyldimethylsilylmethyl-2'-propen-1'-yl)-1-

oxaspiro[2,4]heptane (16). Colorless oil, (65\%); IR (neat): 3050, $1625 \mathrm{~cm}^{-1} ;{ }^{1} \mathrm{H}$ NMR (300 $\left.\mathrm{MHz}_{\mathrm{CDCl}}, \delta\right): 7.52-7.14(\mathrm{~m}, 10 \mathrm{H}), 4.88(\mathrm{~s}, 1 \mathrm{H}), 4.63(\mathrm{~s}, 1 \mathrm{H}), 3.08(\mathrm{~d}, J=11 \mathrm{~Hz}, 1 \mathrm{H}), 2.69$ $2.60(\mathrm{~m}, 1 \mathrm{H}), 2.27(\mathrm{~d}, J=5 \mathrm{~Hz}, 1 \mathrm{H}), 2.12-1.92(\mathrm{~m}, 2 \mathrm{H}), 1.78-1.71(\mathrm{~m}, 1 \mathrm{H}), 1.66(\mathrm{~d}, J=14.2 \mathrm{~Hz}$, $1 \mathrm{H}), 1.51(\mathrm{~d}, J=5 \mathrm{~Hz}, 1 \mathrm{H}), 159-1.48(\mathrm{~m}, 4 \mathrm{H}), 0.22\left(\mathrm{~s}, 3 \mathrm{H}, \mathrm{CH}_{3} \mathrm{Si}\right), 0.20\left(\mathrm{~s}, 3 \mathrm{H}, \mathrm{CH}_{3} \mathrm{Si}\right) ;{ }^{13} \mathrm{C}$ NMR $\left(\mathrm{CDCl}_{3}, 75 \mathrm{MHz}, \delta\right): 148.8,142.5,139.2,133.6,128.9,128.7,127.7,126.1,108.7,65.7$, 53.6, 49.8, 42.4, 34.2, 32.1, 23.7, 21.7, -2.6, -2.9.

\section{[4R*,1'R*]-4-(1'-Phenyl-2'-phenyldimethylsilylmethyl-2'-propen-1'-yl)-1-}

oxaspiro[2,5]octane (17). Colorless oil, (67\%); IR (neat): 3066, 1629, 1248, 1112, $874 \mathrm{~cm}^{-1} ;{ }^{1} \mathrm{H}$ NMR (300 MHz, $\left.\mathrm{CDCl}_{3}, \delta\right): 7.56-7.25(\mathrm{~m}, 10 \mathrm{H}), 4.96(\mathrm{~s}, 1 \mathrm{H}), 4.75(\mathrm{~s}, 1 \mathrm{H}), 3.36(\mathrm{~d}, J=11.4 \mathrm{~Hz}$, $1 \mathrm{H}), 2.36(\mathrm{~d}, J=4.6 \mathrm{~Hz}, 1 \mathrm{H}), 2.26(\mathrm{~d}, J=4.6 \mathrm{~Hz}, 1 \mathrm{H}), 2.25-2.15(\mathrm{~m}, 1 \mathrm{H}), 1.90-1.40(\mathrm{~m}, 8 \mathrm{H})$, $1.62(\mathrm{~s}, 2 \mathrm{H}), 0.22\left(\mathrm{~s}, 3 \mathrm{H}, \mathrm{CH}_{3} \mathrm{Si}\right), 0.16\left(\mathrm{~s}, 3 \mathrm{H}, \mathrm{CH}_{3} \mathrm{Si}\right) ;{ }^{13} \mathrm{C} \mathrm{NMR}\left(\mathrm{CDCl}_{3}, 75 \mathrm{MHz}, \delta\right): 147.3$, $142.5,139.4,133.7,129.0,128.6,128.1,127.8,126.3,110.0,60.6,53.7,52.5,41.2,33.3,29.2$, 25.2, 23.3, 22.3, -2.4, -3.1; Anal. Calcd. for $\mathrm{C}_{25} \mathrm{H}_{32} \mathrm{OSi}$ : C, 79.73; H, 8.56. Found: C, 79.98; H, 8.77 .

[4S*,7R*]-4-(3'-Phenyldimethylsilylmethyl-2'-methylbut-3'-en-2-yl)-7-methyl-1-

oxaspiro[2,5]octane (18). Colorless oil, (70\%). [ $\alpha]^{20}{ }_{\mathrm{D}}-20.4$ (c 1..32, $\mathrm{HCCl}_{3}$ ); IR (neat): 1624, $1248 \mathrm{~cm}^{-1} ;{ }^{1} \mathrm{H}$ NMR $\left(\mathrm{CDCl}_{3}, 300 \mathrm{MHz}, \delta\right): 7.62-7.43(\mathrm{~m}, 5 \mathrm{H}), 4.88(\mathrm{~s}, 1 \mathrm{H}), 4.70(\mathrm{~s}, 1 \mathrm{H}), 3.43(\mathrm{~d}$, $J=4.5 \mathrm{~Hz}, 1 \mathrm{H}), 2.37(\mathrm{~d}, J=4.5 \mathrm{~Hz}, 1 \mathrm{H}), 1.95-1.84(\mathrm{~m}, 3 \mathrm{H}), 1.80(\mathrm{dd}, J=12.5$ and $2.9 \mathrm{~Hz}, 1 \mathrm{H})$, $1.75(\mathrm{~s}, 2 \mathrm{H}), 1.59$ (dd, $J=12.5$ and $2.9 \mathrm{~Hz}, 1 \mathrm{H}), 1.51$ (broad d, $J=12.7 \mathrm{~Hz}, 1 \mathrm{H}), 1.14-1.04(\mathrm{~m}$, 2H), $0.98(\mathrm{~s}, 3 \mathrm{H}), 0.92(\mathrm{~d}, J=7.5 \mathrm{~Hz}, 3 \mathrm{H}), 0.91(\mathrm{~s}, 3 \mathrm{H}), 0.40(\mathrm{~s}, 3 \mathrm{H}), 0.39(\mathrm{~s}, 3 \mathrm{H}) ;{ }^{13} \mathrm{C}$ NMR $\left(\mathrm{CDCl}_{3}, 75 \mathrm{MHz}, \delta\right): 155.6,139.6,133.6$ 128.9, 127.8, 109.0, 62.0, 54.2, 46.1, 44.2, 41.5, 35.8, 31.0, 29.3, 25.6, 22.1 21.6, 19.7, -1.8, -1.9; Anal. Calcd. for $\mathrm{C}_{22} \mathrm{H}_{34} \mathrm{OSi}$ : C, 77.13; H, 10.00 . Found: C, 77.39; H, 10.21. 
[4R*,1'R*]-4-(1'-Phenyl-2'-phenyldimethylsilylmethyl-2'-propen-1'-yl)-1-

oxaspiro[2,6]nonane (19). Colorless oil, (65\%); IR (neat): $1638 \mathrm{~cm}^{-1}$; ${ }^{1} \mathrm{H} \mathrm{NMR}(300 \mathrm{MHz}$, $\left.\mathrm{CDCl}_{3}, \delta\right): 7.51-7.20(\mathrm{~m}, 10 \mathrm{H}), 4.89(\mathrm{~s}, 1 \mathrm{H}), 4.67(\mathrm{~s}, 1 \mathrm{H}), 3.17(\mathrm{~d}, J=11.5 \mathrm{~Hz}, 1 \mathrm{H}), 2.42(\mathrm{~d}, J=$ $4.7 \mathrm{~Hz}, 1 \mathrm{H}), 2.29$ (d, $J=4.7 \mathrm{~Hz}, 1 \mathrm{H}), 2.22-2.15(\mathrm{~m}, 1 \mathrm{H}), 1.75-1.40(\mathrm{~m}, 10 \mathrm{H}), 1.61(\mathrm{~d}, J=15.1$ $\mathrm{Hz}, 1 \mathrm{H}), 1.53(\mathrm{~d}, J=15.1 \mathrm{~Hz}, 1 \mathrm{H}), 0.15(\mathrm{~s}, 3 \mathrm{H}), 0.12(\mathrm{~s}, 3 \mathrm{H}) ;{ }^{13} \mathrm{C} \mathrm{NMR}\left(\mathrm{CDCl}_{3}, 75 \mathrm{MHz}, \delta\right)$ : 147.5 , 142.2, 139.4, 133.6, 128.9, 127.8, 127.7, 126.2, 109.8, 60.9, 56.0, 53.9, 42.8, 34.4, 30.2, 27.3, 26.6, 25.5, 22.1, -2.4, -3.2; Anal. Calcd. for $\mathrm{C}_{26} \mathrm{H}_{34} \mathrm{OSi}$ : C, 79.94; H, 8.77. Found: C, 80.21; H, 9.01 .

[4R*,1'S*]-4-(1'-Phenyl-2'-phenyldimethylsilylmethyl-2'-propen-1'-yl)-1-

oxaspiro[2,6]nonane (20). Colorless oil, (67\%); IR (neat): 1640, $1248 \mathrm{~cm}^{-1} ;{ }^{1} \mathrm{H}$ NMR (300 $\left.\mathrm{MHz}, \mathrm{CDCl}_{3}, \delta\right): 7.45-7.15(\mathrm{~m}, 10 \mathrm{H}), 5.01(\mathrm{~s}, 1 \mathrm{H}), 4.76(\mathrm{~s}, 1 \mathrm{H}), 3.11(\mathrm{~d}, J=10.2 \mathrm{~Hz}, 1 \mathrm{H}), 2.57$ $(\mathrm{d}, J=4.8 \mathrm{~Hz}, 1 \mathrm{H}), 2.52(\mathrm{~d}, J=4.8 \mathrm{~Hz}, 1 \mathrm{H}), 2.21(\mathrm{ddd}, J=10.2,8.9$ and $4.3 \mathrm{~Hz}, 1 \mathrm{H}), 1.73(\mathrm{~d}, J$ $=14.7 \mathrm{~Hz}, 1 \mathrm{H}), 1.69-1.47(\mathrm{~m}, 7 \mathrm{H}), 1.58(\mathrm{~d}, J=14.7 \mathrm{~Hz}, 1 \mathrm{H}), 1.40-1.22(\mathrm{~m}, 3 \mathrm{H}), 0.15(\mathrm{~s}, 3 \mathrm{H})$, 0.12 (s, 3H); ${ }^{13} \mathrm{C} \mathrm{NMR}\left(\mathrm{CDCl}_{3}, 75 \mathrm{MHz}, \delta\right): 148.3,142.0,139.4,133.5,129.1,128.8,128.1$, $127.6,126.3,109.4,61.5,55.4,55.1,43.6,35.0,29.2,27.0,26.8,25.8,24.3,-2.5,-2.9$.

\section{Cyclization of epoxyallylsilanes 15-20. Typical procedure ${ }^{12}$}

$\mathrm{BF}_{3} . \mathrm{OEt}_{2}(1.2 \mathrm{mmol})$ was slowly added to a solution of the epoxyallylsilane $(1 \mathrm{mmol})$ in DCM $(10 \mathrm{ml})$ under nitrogen at $0^{\circ} \mathrm{C}$. After stirring for $40 \mathrm{~min}$ at this temperature brine was added and the mixture extracted with ether. The organic layer was dried over $\mathrm{MgSO}_{4}$, the solvent was evaporated and the residue purified by chromatography to give the corresponding methylenecyclohexanols 21-29 (Table 2).

[3a' $R^{*}, 7$ ' $S^{*}, 7 a^{\prime} S^{*}$ ]-5'-Methyleneoctahydrospiro[cyclopentane-1,4'-inden]-7'-ol

(21). Colorless oil, (89\%); IR (neat): 3622, 3373, 1640, 1039, $887 \mathrm{~cm}^{-1} ;{ }^{1} \mathrm{H} \mathrm{NMR}\left(300 \mathrm{MHz}, \mathrm{CDCl}_{3}\right.$, $\delta): 4.83(\mathrm{~s}, 1 \mathrm{H}), 4.70(\mathrm{~s}, 1 \mathrm{H}), 3.34(\mathrm{td}, J=11.6$ and $4.9 \mathrm{~Hz}, 1 \mathrm{H}), 2.42(\mathrm{dd}, J=12.2$ and $5.0 \mathrm{~Hz}$, $1 \mathrm{H}), 2.23(\mathrm{t}, J=11.6 \mathrm{~Hz}, 1 \mathrm{H}), 1.92-1.80(\mathrm{~m}, 4 \mathrm{H}), 1.79-1.50(\mathrm{~m}, 11 \mathrm{H}), 1.45-1.32(\mathrm{~m}, 1 \mathrm{H}), 1.29$ $1.15(\mathrm{~m}, 1 \mathrm{H}) ;{ }^{13} \mathrm{C} \mathrm{NMR}\left(\mathrm{CDCl}_{3}, 75 \mathrm{MHz}, \delta\right): 149.4(\mathrm{C}), 108.9\left(\mathrm{CH}_{2}\right), 71.6(\mathrm{CH}), 51.8(\mathrm{CH})$, $50.6(\mathrm{C}), 46.9(\mathrm{CH}), 42.5\left(\mathrm{CH}_{2}\right), 38.9\left(\mathrm{CH}_{2}\right), 34.3\left(\mathrm{CH}_{2}\right), 27.9\left(\mathrm{CH}_{2}\right), 25.4\left(\mathrm{CH}_{2}\right), 23.8\left(\mathrm{CH}_{2}\right)$, $23.3\left(\mathrm{CH}_{2}\right), 21.2\left(\mathrm{CH}_{2}\right)$; Anal. Calcd. for $\mathrm{C}_{14} \mathrm{H}_{22} \mathrm{O}: \mathrm{C}, 81.50 ; \mathrm{H}, 10.75$. Found: C, 81.79; $\mathrm{H}$, 10.97 .

[3a $\left.S^{*}, 4 S, 7 R^{*}, 7 \mathrm{a} S^{*}\right]-6-M e t h y l e n e-7-p h e n y l o c t a h y d r o-1 H$-inden-4-ol (22). White solid, (85\%). m.p. 80.5-81.3 ${ }^{\circ} \mathrm{C}$; IR (neat): $3627,3422,1650,903 \mathrm{~cm}^{-1} ;{ }^{1} \mathrm{H} \mathrm{NMR}\left(300 \mathrm{MHz}, \mathrm{CDCl}_{3}, \delta\right)$ : 7.34$7.21(\mathrm{~m}, 5 \mathrm{H}), 4.96(\mathrm{~s}, 1 \mathrm{H}), 4.94(\mathrm{~s}, 1 \mathrm{H}), 3.75(\mathrm{~d}, J=4 \mathrm{~Hz}, 1 \mathrm{H}), 3.57-345(\mathrm{~m}, 1 \mathrm{H}), 2.75(\mathrm{dt}, J=$ 13.5 and $5.1 \mathrm{~Hz}, 1 \mathrm{H}), 2.57(\mathrm{td}, J=13.5$ and $2.0 \mathrm{~Hz}, 1 \mathrm{H}), 2.02-1.87(\mathrm{~m}, 1 \mathrm{H}), 1.86-1.54(\mathrm{~m}, 6 \mathrm{H})$, 1.32-1.20 (m, 1H), 1.12-1.00 (m, 1H); ${ }^{13} \mathrm{C} \mathrm{NMR}\left(\mathrm{CDCl}_{3}, 75 \mathrm{MHz}, \delta\right): 149.7$ (C), $142.8(\mathrm{C})$, $130.3(\mathrm{CH}), 129,2(\mathrm{CH}), 127.3(\mathrm{CH}), 115.0\left(\mathrm{CH}_{2}\right), 77.5(\mathrm{CH}), 52.1(\mathrm{CH}), 48.9(\mathrm{CH}), 47.3(\mathrm{CH})$, $44.5\left(\mathrm{CH}_{2}\right), 29.3\left(\mathrm{CH}_{2}\right), 29.1\left(\mathrm{CH}_{2}\right), 23.4\left(\mathrm{CH}_{2}\right)$; Anal. Calcd. for $\mathrm{C}_{16} \mathrm{H}_{20} \mathrm{O}: \mathrm{C}, 84.16 ; \mathrm{H}, 8.83$. Found: C, 84.40; H, 9.07.

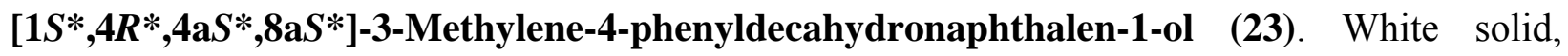
(78\%). m.p. 107.3-108.1 ${ }^{\circ} \mathrm{C}$; IR (neat): 3626, 3367, 1644, $902 \mathrm{~cm}^{-1} ;{ }^{1} \mathrm{H} \mathrm{NMR} \mathrm{(300} \mathrm{MHz,} \mathrm{CDCl}_{3}$, 


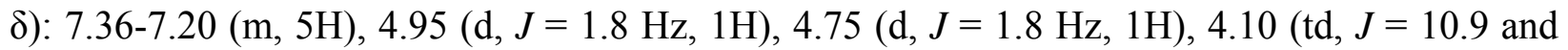
$5.1 \mathrm{~Hz}, 1 \mathrm{H}), 3.53(\mathrm{~d}, J=3.0 \mathrm{~Hz}, 1 \mathrm{H}), 2.84(\mathrm{dd}, J=12.8$ and $5.1 \mathrm{~Hz}, 1 \mathrm{H}), 2.23(\mathrm{dd}, J=12.8$ and $10.9 \mathrm{~Hz}, 1 \mathrm{H}), 2.17(\mathrm{dd}, J=10.4$ and $2.4 \mathrm{~Hz}, 1 \mathrm{H}), 1.95(\mathrm{dq}, J=12.6$ and $4.1 \mathrm{~Hz}, 1 \mathrm{H}), 1.90-1.82$ $(\mathrm{m}, 1 \mathrm{H}), 1.81-1.73(\mathrm{~m}, 1 \mathrm{H}), 1.71-1.35(\mathrm{~m}, 5 \mathrm{H}), 1.23(\mathrm{dd}, \mathrm{J}=12.6$ and $3.5 \mathrm{~Hz}, 1 \mathrm{H}), 1.22-1.10(\mathrm{~m}$, $1 \mathrm{H}) ;{ }^{13} \mathrm{C} \mathrm{NMR}\left(\mathrm{CDCl}_{3}, 75 \mathrm{MHz}, \delta\right): 144.6(\mathrm{C}), 140.0(\mathrm{C}), 130.6(\mathrm{CH}), 127.7(\mathrm{CH}), 126.6(\mathrm{CH})$, $110.9\left(\mathrm{CH}_{2}\right), 66.8(\mathrm{CH}), 55.0(\mathrm{CH}), 45.9\left(\mathrm{CH}_{2}\right), 45.3(\mathrm{CH}), 43.6(\mathrm{CH}), 26.6\left(\mathrm{CH}_{2}\right) 26.2\left(\mathrm{CH}_{2}\right)$, $22.7\left(\mathrm{CH}_{2}\right), 20.4\left(\mathrm{CH}_{2}\right)$; Anal. Calcd. for $\mathrm{C}_{17} \mathrm{H}_{22} \mathrm{O}$ : C, 84.25; H, 9.15. Found: C, 84.60; H, 9.44.

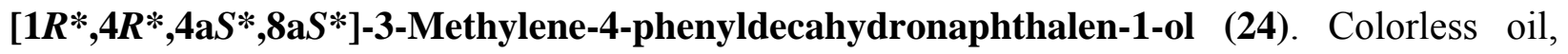
(10\%); IR (neat): 3569, 3460, 1640, 1068, $883 \mathrm{~cm}^{-1} ;{ }^{1} \mathrm{H} \mathrm{NMR}\left(300 \mathrm{MHz}, \mathrm{CDCl}_{3}, \delta\right): 7.47-7.19$ $(\mathrm{m}, 5 \mathrm{H}), 5.06(\mathrm{~s}, 1 \mathrm{H}), 4.81(\mathrm{~s}, 1 \mathrm{H}), 3.98-3.91(\mathrm{~m}, 1 \mathrm{H}), 3.54(\mathrm{~d}, J=4.5 \mathrm{~Hz}, 1 \mathrm{H}), 2.83(\mathrm{ddd}, J=$ $14.3,3.5$ and $1.6 \mathrm{~Hz}, 1 \mathrm{H}), 2.43(\mathrm{dd}, J=14.3$ and $3.1 \mathrm{~Hz}, 1 \mathrm{H}), 1.92-1.82(\mathrm{~m}, 2 \mathrm{H}), 1.80-1.50(\mathrm{~m}$, $4 \mathrm{H}), 1.49-1.39(\mathrm{~m}, 2 \mathrm{H}), 1.29-1.15(\mathrm{~m}, 2 \mathrm{H}), 0.98-0.81(\mathrm{~m}, 1 \mathrm{H}) ;{ }^{13} \mathrm{C} \mathrm{NMR}\left(\mathrm{CDCl}_{3}, 75 \mathrm{MHz}, \delta\right)$ : $146.7(\mathrm{C}), 142.4(\mathrm{C}), 128.7(\mathrm{CH}), 128.2(\mathrm{CH}), 126.2(\mathrm{CH}), 113.5\left(\mathrm{CH}_{2}\right), 71.1(\mathrm{CH}), 54.7(\mathrm{CH})$, $41.5(\mathrm{CH}), 39.9\left(\mathrm{CH}_{2}\right), 39.4(\mathrm{CH}), 30.8\left(\mathrm{CH}_{2}\right), 29.9\left(\mathrm{CH}_{2}\right), 26.2\left(\mathrm{CH}_{2}\right), 24.2\left(\mathrm{CH}_{2}\right)$.

[1S,4aR,7R,8aS]-4,4,7-Trimethyl-3-methylenedecahydronaphthalen-1-ol (25). Colorless oil, (88\%). $[\alpha]^{20}{ }_{\mathrm{D}}+15.3$ (c $0.62, \mathrm{HCCl}_{3}$ ); IR (neat): 3331, 1640, 1048, $889 \mathrm{~cm}^{-1} ;{ }^{1} \mathrm{H}$ NMR (300 MHz, $\left.\mathrm{CDCl}_{3}, \delta\right): 4.78(\mathrm{t}, J=1.6 \mathrm{~Hz}, 1 \mathrm{H}), 4.64(\mathrm{t}, J=1.6 \mathrm{~Hz}, 1 \mathrm{H}), 3.82(\mathrm{td}, J=11.1$ and $5.5 \mathrm{~Hz}, 1 \mathrm{H})$, $2.49(\mathrm{dd}, J=12.7$ and $5.5 \mathrm{~Hz}, 1 \mathrm{H}), 2.33(\mathrm{dd}, J=12.7$ and $11.1 \mathrm{~Hz}, 1 \mathrm{H}), 2.15-2.04(\mathrm{~m}, 2 \mathrm{H}), 1.78-$ $1.46(\mathrm{~m}, 5 \mathrm{H}), 1.36(\mathrm{dt}, J=12.5$ and $4.1 \mathrm{~Hz}, 1 \mathrm{H}), 1.18(\mathrm{~s}, 3 \mathrm{H}), 1.04(\mathrm{~s}, 3 \mathrm{H}), 1.03-0.94(\mathrm{~m}, 2 \mathrm{H})$, $0.85(\mathrm{~d}, J=6.5 \mathrm{~Hz}, 3 \mathrm{H}) ;{ }^{13} \mathrm{C} \mathrm{NMR}\left(\mathrm{CDCl}_{3}, 75 \mathrm{MHz}, \delta\right): 151.5(\mathrm{C}), 108.6\left(\mathrm{CH}_{2}\right), 68.9(\mathrm{CH}), 48.4$ $(\mathrm{CH}), 42.1\left(\mathrm{CH}_{2}\right), 40.0(\mathrm{CH}), 39.4(\mathrm{C}), 35.5\left(\mathrm{CH}_{2}\right), 35.4\left(\mathrm{CH}_{2}\right), 28.6\left(\mathrm{CH}_{3}\right), 25.9(\mathrm{CH}), 25.5$ $\left(\mathrm{CH}_{3}\right)$, $23.4\left(\mathrm{CH}_{2}\right), 22.7\left(\mathrm{CH}_{3}\right)$; Anal. Calcd. for $\mathrm{C}_{14} \mathrm{H}_{24} \mathrm{O}: \mathrm{C}, 80.71 ; \mathrm{H}, 11.61$. Found: $\mathrm{C}, 81.01$; $\mathrm{H}, 11.86$.

[1S*,4a $\left.R^{*}, 9 \mathrm{a} S^{*}\right]-3-($ Phenyldimethylsilylmethyl)-4-phenyl-2,4a,5,6,7,8,9,9a-octahydro-1Hbenzo[7]annulen-1-ol (26). Colorless oil, (64\%); IR (neat): 3627, 3450, 1644, 1548, 1248, 1111 $\mathrm{cm}^{-1}$; ${ }^{1} \mathrm{H}$ NMR $\left(300 \mathrm{MHz}, \mathrm{CDCl}_{3}, \delta\right): 7.40-7.20(\mathrm{~m}, 8 \mathrm{H}), 7.03-6.90(\mathrm{~m}, 2 \mathrm{H}), 3.77$ (td, $J=8.0$ and $4.8 \mathrm{~Hz}, 1 \mathrm{H}), 2.72-2.64(\mathrm{~m}, 1 \mathrm{H}), 2.36(\mathrm{dd}, J=16.2$ and $4.8 \mathrm{~Hz}, 1 \mathrm{H}), 1.95(\mathrm{dd}, J=16.2$ and 8.0 $\mathrm{Hz}, 1 \mathrm{H}), 1.87$ (d, $J=14.0 \mathrm{~Hz}, 1 \mathrm{H}), 1.86-1.72(\mathrm{~m}, 3 \mathrm{H}), 1.70-1.51(\mathrm{~m}, 4 \mathrm{H}), 1.46(\mathrm{~d}, J=14.0 \mathrm{~Hz}$, $1 \mathrm{H}), 1.45-1.33(\mathrm{~m}, 3 \mathrm{H}), 1.27-1.02(\mathrm{~m}, 2 \mathrm{H}), 0.25(\mathrm{~s}, 3 \mathrm{H}), 0.19(\mathrm{~s}, 3 \mathrm{H}) ;{ }^{13} \mathrm{C} \mathrm{NMR}\left(\mathrm{CDCl}_{3}, 75\right.$ $\mathrm{MHz}, \delta): 142.8(\mathrm{C}), 139.3(\mathrm{C}), 134.7(\mathrm{C}), 133.5(\mathrm{CH}), 129.5(\mathrm{CH}), 128.8(\mathrm{CH}), 127.8(\mathrm{CH})$, $127.6(\mathrm{CH}), 126.9(\mathrm{C}), 125.8(\mathrm{CH}), 70.5(\mathrm{CH}), 44.4(\mathrm{CH}), 43.9(\mathrm{CH}), 40.1\left(\mathrm{CH}_{2}\right), 31.0\left(\mathrm{CH}_{2}\right)$, $29.3\left(\mathrm{CH}_{2}\right), 29.0\left(\mathrm{CH}_{2}\right), 28.5\left(\mathrm{CH}_{2}\right), 25.5\left(\mathrm{CH}_{2}\right), 23.9\left(\mathrm{CH}_{2}\right),-1.9\left(2 \mathrm{xCH}_{3}\right)$; Anal. Calcd. for $\mathrm{C}_{26} \mathrm{H}_{34} \mathrm{OSi}$ : C, 79.94; H, 8.77. Found: C, 80.21; H, 8.90.

[1 $\left.\boldsymbol{R}^{*}, \mathbf{4} \boldsymbol{R}^{*}, 4 \mathrm{a} S^{*}, \mathbf{9 a} S^{*}\right]-3-M e t h y l e n e-4-p h e n y l d e c a h y d r o-1 H$-benzo[7]annulen-1-ol (27). Colorless oil, (16\%); IR (neat): 3620, 3397, 1646, $1033 \mathrm{~cm}^{-1} ;{ }^{1} \mathrm{H}$ NMR (300 MHz, $\mathrm{CDCl}_{3}, \delta$ ): 7.40-7.20 (m, 5H), $5.05(\mathrm{~s}, 1 \mathrm{H}), 4.81(\mathrm{~s}, 1 \mathrm{H}), 3.90(\mathrm{dd}, J=6.2$ and $3.1 \mathrm{~Hz}, 1 \mathrm{H}), 3.62(\mathrm{~d}, J=4.2$ $\mathrm{Hz}, 1 \mathrm{H}), 2.65-2.55(\mathrm{~m}, 2 \mathrm{H}), 2.41(\mathrm{dt}, J=9.4$ and $4.2 \mathrm{~Hz}, 1 \mathrm{H}), 2.01(\mathrm{dq}, J=10.2$ and $2.7 \mathrm{~Hz}$,

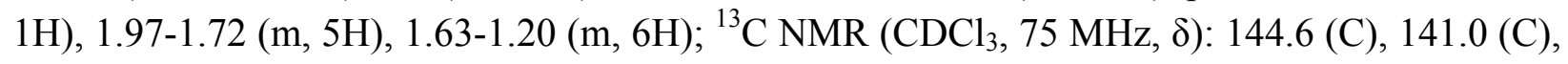
129.4 (CH), $127.8(\mathrm{CH}), 125.8(\mathrm{CH}), 112.0\left(\mathrm{CH}_{2}\right), 72.2(\mathrm{CH}), 53.5(\mathrm{CH}), 45.9\left(\mathrm{CH}_{2}\right), 43.9(\mathrm{CH})$, $42.7(\mathrm{CH}), 31.7\left(\mathrm{CH}_{2}\right), 29.8\left(\mathrm{CH}_{2}\right), 29.2\left(\mathrm{CH}_{2}\right), 25.0\left(\mathrm{CH}_{2}\right), 24.6\left(\mathrm{CH}_{2}\right)$. 


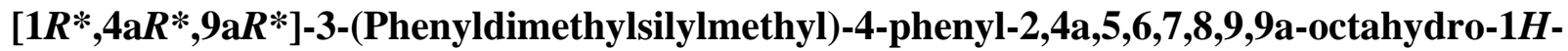
benzo[7]annulen-1-ol (28). Colorless oil, (38\%); IR (neat): 3627, 3420, 1456, $903 \mathrm{~cm}^{-1} ;{ }^{1} \mathrm{H}$ NMR (300 MHz, $\left.\mathrm{CDCl}_{3}, \delta\right): 7.37-7.20(\mathrm{~m}, 8 \mathrm{H}), 6.96-6.90(\mathrm{~m}, 2 \mathrm{H}), 3.92(\mathrm{dt}, J=8.6$ and $4.2 \mathrm{~Hz}$, $1 \mathrm{H}), 2.75(\mathrm{dt}, J=12.3$ and $4.6 \mathrm{~Hz}, 1 \mathrm{H}), 2.20-2.03(\mathrm{~m}, 2 \mathrm{H}), 1.99-1.90(\mathrm{~m}, 1 \mathrm{H}), 1.83-1.70(\mathrm{~m}$, $3 \mathrm{H}), 1.68-1.05(\mathrm{~m}, 9 \mathrm{H}), 0.92-0.83(\mathrm{~m}, 1 \mathrm{H}), 0.23(\mathrm{~s}, 3 \mathrm{H}), 0.17(\mathrm{~s}, 3 \mathrm{H}) ;{ }^{13} \mathrm{C} \mathrm{NMR}\left(\mathrm{CDCl}_{3}, 75\right.$ $\mathrm{MHz}, \delta): 142.5(\mathrm{C}), 139.3(\mathrm{C}), 134.0(\mathrm{C}), 133.5(\mathrm{CH}), 129.4(\mathrm{CH}), 128.8(\mathrm{CH}), 127.8(\mathrm{CH})$, 127.6 (CH), $127.3(\mathrm{C}), 125.7(\mathrm{CH}), 70.6(\mathrm{CH}), 44.2(\mathrm{CH}) ; 43.7(\mathrm{CH}), 37.4\left(\mathrm{CH}_{2}\right), 32.2\left(\mathrm{CH}_{2}\right)$, $31.4\left(\mathrm{CH}_{2}\right), 29.4\left(\mathrm{CH}_{2}\right), 25.6\left(\mathrm{CH}_{2}\right), 24.1\left(\mathrm{CH}_{2}\right), 22.7\left(\mathrm{CH}_{2}\right),-1.9\left(2 \mathrm{xCH}_{3}\right)$; Anal. Calcd. for $\mathrm{C}_{26} \mathrm{H}_{34} \mathrm{OSi}$ : C, 79.94; H, 8.77. Found: C, 80.30; H, 8.98.

[1S*,4S*,4a $\left.S^{*}, 9 \mathrm{a} R^{*}\right]-3-M e t h y l e n e-4-p h e n y l d e c a h y d r o-1 H$-benzo[7]annulen-1-ol

(29).

Colorless oil, (37\%); IR (neat): 3579, 3441, 1641, $1005 \mathrm{~cm}^{-1} ;{ }^{1} \mathrm{H}$ NMR (300 MHz, $\left.\mathrm{CDCl}_{3}, \delta\right)$ : 7.32-7.10 (m, 5H), $4.82(\mathrm{~s}, 1 \mathrm{H}), 4.14(\mathrm{~s}, 1 \mathrm{H}), 3.94-3.90(\mathrm{~m}, 1 \mathrm{H}), 2.96(\mathrm{~d}, J=11.5 \mathrm{~Hz}, 1 \mathrm{H}), 2.54$ $(\mathrm{s}, 2 \mathrm{H}), 1.93(\mathrm{qt}, J=11.0$ and $2.4 \mathrm{~Hz}, 1 \mathrm{H}), 1.85-1.70(\mathrm{~m}, 3 \mathrm{H}), 1.60-1.50(\mathrm{~m}, 6 \mathrm{H}), 1.45-1.10(\mathrm{~m}$, $3 \mathrm{H}) ;{ }^{13} \mathrm{C} \mathrm{NMR}\left(\mathrm{CDCl}_{3}, 75 \mathrm{MHz}, \delta\right): 147.9(\mathrm{C}), 141.8(\mathrm{C}), 129.3(\mathrm{CH}), 128.2(\mathrm{CH}), 126.2(\mathrm{CH})$, $113.6\left(\mathrm{CH}_{2}\right), 73.4(\mathrm{CH}), 56.4(\mathrm{CH}), 49.2(\mathrm{CH}), 44.1\left(\mathrm{CH}_{2}\right), 43.3(\mathrm{CH}), 32.7\left(\mathrm{CH}_{2}\right), 32.5\left(\mathrm{CH}_{2}\right)$, $27.6\left(\mathrm{CH}_{2}\right), 25.6\left(\mathrm{CH}_{2}\right), 25.3\left(\mathrm{CH}_{2}\right)$; Anal. Calcd. for $\mathrm{C}_{18} \mathrm{H}_{24} \mathrm{O}: \mathrm{C}, 84.32 ; \mathrm{H}, 9.44$. Found: $\mathrm{C}$, 84.70; H, 9.73.

\section{Acknowledgements}

This work was financially supported by and the Spanish "Ministerio de Ciencia y Tecnología"(project CTQ2006-02436/BQU) and the "Junta de Castilla y León" (project VA074A08)

\section{References}

1. (a) Fleming, I. In Comprehensive Organic Synthesis; Trost, B. M.; Fleming, I. Eds; Pergamon: Oxford. U.K. 1991, Vol 2. (b) Fleming, I. Science of Synthesis: Houben-Weyl Methods of Molecular Transformations; Thieme: Stuttgart, 2001, Vol. 4.

2. (a) Barbero, A.; Pulido, F. J. Acc. Chem. Res. 2004, 37, 817. (b) Barbero, A.; Pulido, F. J. Synthesis (Special Topic) 2004, 779. (c) Schinzer, D. Chem. Rev. 1995, 95, 1375.

3. (a) Fleming, I.; Pulido, F. J. Chem. Commun. 1986, 1010. (b) Fleming, I.; Pulido, F. J.; Rowley, M.; Cuadrado, P.; González, A. M. Tetrahedron (Symposia-in-Print) 1989, 45, 413.

(c) Krause, N. Modern Organocopper Chemistry; Wiley-VCH: Dortmund, Germany, 2002, Cap. 3.

4. Barbero, A.; García, C.; Pulido, F. J. Tetrahedron (Symposia-in-Print) 2000, 56, 2739.

5. (a) Liepins, V.; Karlström, S. E.; Bäckvall, J.E. Org. Lett. 2000, 2, 1237. (b) Barbero, A.; Castreño, P.; García, C.; Pulido, F. J. J. Org. Chem., 2001, 66, 7723. (c) Barbero, A.; Blanco, 
Y.; Pulido, F. J. Chem. Commun., 2001, 1606. (d) Barbero, A.; Castreño P.; Pulido, F. J. J. Am. Chem. Soc. 2005, 127, 8022. (e) Barbero, A.; Blanco, Y.; F. J. Pulido, F. J. J. Org. Chem. 2005, 70, 6876.

6. (a) Barbero, A.; Pulido, F. J.; Sañudo, M. C. Beilstein J. Org. Chem. 2007, 3, 16. (b) Pulido, F. J.; Barbero, A. Silyl and stannyl derivatives of organocopper compounds In The Patai Series: The Chemistry of Organocopper Compounds, Z. Rappoport, Z.; Marek, I. Eds., Part II, Chap. 16, John Wiley \& Sons: Chichester, U.K., 2009 (in press).

7. (a) Beszant, S.; Giannini, E.; Zanoni, G.; Vidari, G. Tetrahedron: Asymmetry 2002, 13, 1245.

(b) Overman, L. E.; Renhowe, P. A. J. Org. Chem. 1994, 59, 4138.

8. (a) Molander, G. A.; Andrews, S. W. J. Org. Chem. 1989, 54, 3114. (b) Sugita, Y.; Kimura, Y.; Yokoe, I. Tetrahedron Lett. 1999, 40, 5877.

9. (a) Nishitani, K.; Harada, Y.; Nakamura, Y.; Yokoo, K.; Yamakawa, K. Tetrahedron Lett. 1994, 35, 7809.

10. Jung, M. E.; Cho, Y. M.; Jung, Y. H. Tetrahedron Lett. 1996, 37, 3.

11. (a) Corey, E. J.; Staas, D. D. J. Am. Chem. Soc. 1998, 120, 3526. (b) Molander, G. A.; Shubert, D. C. J. Am. Chem. Soc. 1987, 109, 576.12.

12. Barbero, A.; Castreño P.; Pulido, F. J. Org. Lett. 2003, 5, 4045.

13. Barbero, A.; Castreño P.; Fernández, G.; Pulido, F. J. J. Org. Chem. 2005, 70, 10747.

14. Barbero, A.; Cuadrado, P.; González, A. M.; Pulido, F. J.; Fleming, I. J. Chem. Soc., Perkin Trans. 1 1991, 2811.

15. Seyferth, D.; Wursthorn, K. R.; Mammarella, R. E. J. Org. Chem. 1977, 42, 3104.

16. Langer, P.; Holtz, E. Synlett 2002, 110.

17. Barbero, A.; Pulido, F. J. Chem. Soc. Rev. 2005, 34, 913.

18. Barbero, A.; Pulido, F. J.; Sañudo, M. C. ARKIVOC 2007 (iv) 220.

19. Structurally similar epoxyallylsilanes bearing the trimethylsilyl group instead of phenyldimethylsilyl group undergo normal cyclization by nucleophilic attack of the allylsilane moiety to the end of the epoxide giving 4-methylene-1-cyclohexanols, as expected. Rearrangement of the epoxide was never observed (see ref. 11).

20. Blanco, F. J.; Cuadrado, P.; González, A. M.; Pulido, F.J.; Fleming, I. Tetrahedron Lett. 1994, 35, 8881.

21. Although we can not be dogmatic about the formation of a single diastereomer, we were not able to isolate or detect other epoxides different from 15-20.

22. Rickborn, B. Comprehensive Organic Synthesis, Pergamon Press: Oxford, Vol. 3, p.733, 1991.

23. Fleming, I.; Newton, T. W.; Roessler, F. J. Chem. Soc., Perkin Trans 1 1981, 2527. 